OPEN ACCESS

Edited by:

Demet Demirkol,

Istanbul University, Turkey

Reviewed by:

Uri Pollak,

Hadassah-Hebrew University Medical

Center, Israe

Danny Eytan,

Technion Israel Institute of

Technology, Israel

*Correspondence:

Yin Zhang

yinzhang9511@163.com

Specialty section

This article was submitted to

Pediatric Critical Care,

a section of the journal

Frontiers in Pediatrics

Received: 09 April 2021

Accepted: 06 July 2021

Published: 03 August 2021

Citation:

Lin J, Zhang Y, Chen M, Dai J,

Song A, Chen J and Tao X (2021) The

Association Between Variability in

Electrolytes and the In-Hospital

Mortality in Critically III Children in

Pediatric Intensive Care Units.

Front. Pediatr. 9:692894.

doi: 10.3389/fped.2021.692894

\section{The Association Between Variability in Electrolytes and the In-Hospital Mortality in Critically III Children in Pediatric Intensive Care Units}

\author{
Jilei Lin ${ }^{1}$, Yin Zhang ${ }^{2 *}$, Meng Chen ${ }^{3}$, Jihong Dai ${ }^{4}$, Anchao Song ${ }^{5}$, Jianchuan Chen ${ }^{4}$ and \\ Xingping Tao ${ }^{6}$ \\ ' Department of Respiratory Medicine, Shanghai Children's Medical Center, Shanghai Jiaotong University School of \\ Medicine, Shanghai, China, ${ }^{2}$ Department of Respiratory and Critical Care Medicine, West China Hospital of Sichuan \\ University, Chengdu, China, ${ }^{3}$ Department of Pediatrics, Meitan People's Hospital, Zunyi, China, ${ }^{4}$ Chongqing Key Laboratory \\ of Pediatrics, Ministry of Education Key Laboratory of Child Development and Disorders, Department of Respiratory Disease, \\ National Clinical Research Center for Child Health and Disorders, Children's Hospital of Chongqing Medical University, \\ Chongqing, China, ${ }^{5}$ School of Public Health and Management, Chongqing Medical University, Chongqing, China, \\ ${ }^{6}$ Department of Pediatrics, Kaiyuan People's Hospital, Kaiyuan, China
}

Objective: This study aimed to explore the association between the variability in electrolytes and the in-hospital mortality in critically ill children admitted into intensive care units (ICUs).

Design: This is a retrospective case-control study.

Setting and Participants: Total of 11,245 children have been admitted to ICUs of Children's Hospital of Zhejiang University from 2010 to 2018.

Methods: The coefficient of variation (CV), standard deviation (SD), and variability independent of the mean (VIM) were calculated as variability indices. High variability was defined as having values in the highest quartile for each parameter. Age, sex, diagnoses of disease, and surgical treatment were adjusted in the multivariable-adjusted logistic regression model.

Results: A total of 11,245 children were included, and 660 patients died in the hospital. The median (P25, P75) potassium, sodium, and chloride of all patients were 3.8 (3.58, 4.09), 136.83 (135.11, 138.60), and 108.67 (105.71, 111.17), respectively. U-shaped relationships between the mean, lowest, and highest levels of potassium, sodium, and chloride and the in-hospital mortality were observed. The lowest mortality was noted when serum potassium, sodium, and chloride were between $\sim 3.5$ and 5.0, 135 and 145, and 105 and $115 \mathrm{mmol} / \mathrm{l}$, respectively. The areas under the curve (AUCs) of three indices of variability in electrolytes were larger than those of the mean and lowest levels of electrolytes in predicting the in-hospital mortality. In the multivariable-adjusted model, the odds ratios and 95\% confidence interval $(\mathrm{Cl})$ of the in-hospital mortality were 3.14 (2.44-4.04) for one parameter, 5.85 (4.54-7.53) for two parameters, and 10.32 (7.81-13.64) for three parameters compared with subjects having no parameters of high variability measured as the CV. The results were consistent when the variability was determined 
using the SD and VIM (all $P$ for trend $<0.001$ ). Consistent results were noted in various subgroup analyses.

Conclusions: This study showed that individuals with higher variability of each parameter were related with higher risk of in-hospital mortality. There was a linear association between the number of high variability parameters and the in-hospital mortality. The variability of electrolytes might be a good predictor for in-hospital mortality of children in ICUs.

Keywords: electrolytes, potassium, sodium, in-hospital mortality, variability, children

\section{INTRODUCTION}

An adequate electrolyte balance is of paramount importance to maintaining physiologic homeostasis and normal metabolism of cells in human body. Internal environment imbalance and electrolyte disorders usually occur in critically ill patients admitted to intensive care units (ICUs), due to different causes. Prompt recognition of electrolyte disorders is essential to the care and treatment of these patients $(1,2)$.

To our knowledge, blood gas analysis is conducted regularly and frequently in critically ill patients in ICUs to monitor patients' physiologic homeostasis. The main items of electrolytes in blood gas analysis are potassium, sodium, and chloride in ICUs. Sodium $\left(\mathrm{Na}^{+}\right)$and chloride $\left(\mathrm{Cl}^{-}\right)$are the major extracellular ions, while potassium $\left(\mathrm{K}^{+}\right)$is the major intracellular cation in the body, and they serve essential functions in the membrane potential and metabolism. ${ }^{1}$ Unbalanced homeostasis will affect neural transmission, vascular tone, etc. and may even lead to life-threatening complications. Dyskalemia is known to induce potentially lethal arrhythmias and cardiac dysfunction, as well as other complications, even mortality risk (3-6). Dysnatremia is also a common finding in ICUs and has been suggested to be a predictor for poor clinical outcomes in patients with different dysfunctions (7-9). Besides, previous studies showed hypo- and hyperchloremia may contribute to higher mortality in patients with major trauma, chronic heart failure, and severe sepsis (10-12). However, it is inappropriate to evaluate the association between serum electrolytes and mortality at a screening visit or using the mean values for pediatric patients with critical illness during the hospitalization, since the serum levels of electrolytes may vary and the normal range of the values is wide in children with different ages. The value of electrolytes may change a lot by the treatment during the hospitalization, so it is inappropriate to use an extreme value to predict the mortality of critically ill children. Therefore, it is important to choose a better way to fit the relationship between electrolytes and inhospital mortality in critically ill children, which can reduce the influence of baseline and extreme values.

Recently, some studies showed that the indices of variability [coefficient of variation (CV), standard deviation (SD) and

\footnotetext{
Abbreviations: $\mathrm{Na}^{+}$, sodium; $\mathrm{Cl}^{-}$, chloride; $\mathrm{K}^{+}$, potassium; AUC, area under the curve; CIs, confidence intervals; CV, coefficient of variation; ICD-10, International Classification of Diseases-10; ICUs, intensive care units; ORs, odds ratios; ROC, receiver operating characteristic; SD, standard deviation; VIM, variability independent of the mean.
}

variability independent of the mean (VIM)], which can reduce the influence of the baseline level of parameters, have emerged as previously unrecognized residual risk factors that is related to the development of various health outcomes (13-15). Therefore, this study aimed to evaluate the association between the variability in electrolytes (including potassium, sodium, and chloride) and in-hospital mortality in critically ill children admitted into ICUs, with a large-scale sample size.

\section{METHODS \\ Study Population}

The data of this observational case-control study were extracted from the Pediatric Intensive Care (PIC) database, a freely accessible pediatric-specific critical care database established by the Children's Hospital of Zhejiang University, a 1,900bed children's hospital in the south of China (16). In this study, patients have been all admitted to ICUs during a period of 8 years from 2010 to 2018. A total of 13,449 admissions were recorded in the PIC database, in which 971 (7.2\%) patients died in hospital. Structured clinical data including the patient demographics, symptoms, vital signs, laboratory results, prescriptions, and surgeries were all collected in the database. The project was approved by the Institutional Review Board of the Children's Hospital, Zhejiang University School of Medicine (Hangzhou, China). The requirement for individual patient consent was waived because the project did not impact clinical care, and all protected health information was deidentified. We accessed the PIC database under a data use agreement from a database manager (Prof. Minhao Li). The results of this study were reported following the Strengthening the Reporting of Observational Studies in Epidemiology statement (17).

\section{Data Extraction}

We included patients if they were admitted to ICUs for the first time and their values of three serum electrolytes (including potassium, sodium, and chloride) were recorded for no less than three times during this hospitalization. However, we excluded patients whose information was seriously absent or wrongly recorded. In this study, the collected data included age, gender, diagnoses of disease (including diseases of the respiratory system, nervous system, circulatory system, digestive system, genitourinary system, and neoplasms), whether surgery was performed or not in this hospital stay, and the length of ICU stay. 
Of note, the International Classification of Diseases-10 (ICD10) code was used to classify diseases of different systems. The endpoint of this study was the in-hospital mortality.

\section{Definitions of Variability Indices}

The in-hospital mortality referred to the rate of death in this hospital stay. Variability in each parameter (including potassium, sodium and, chloride) was defined as variability in their values. We used three indices of variability in this study: CV, SD, and VIM. To explain, the SD was calculated as $\operatorname{sqrt}\left\{\left[\left(x_{1}-x\right)^{\wedge} 2+\right.\right.$ $\left.\left.\left(x_{2}-x\right)^{\wedge} 2+\ldots \ldots\left(x_{n}-x\right)^{\wedge} 2\right] /(n-1)\right\}$; the $\mathrm{CV}$ was calculated as $\mathrm{SD} /$ mean; and the VIM was calculated as $\times \mathrm{SD} / \mathrm{mean}^{\beta}$, where $\beta$ is the regression coefficient, based on the natural logarithm of the SD over the natural logarithm of the mean (18). Specially, each parameter was divided by quartiles, and high variability was defined as values in the highest quartile. The patients were classified further according to the number of high variability parameters using a score ranging from 0 to 3 . In this classification, a score of 0 indicated no high variability parameter, and the scores 1 to 3 indicated the number of high variability parameters among the three serum electrolytes (e.g., a score of 2 indicated high variability in two of the three parameters).

\section{Statistical Analysis}

In this study, continuous variables were presented as means $\pm \mathrm{SD}$. Categorical variables were analyzed by the $\chi^{2}$ test or the Fisher's exact test, as appropriate. They were expressed as numbers $(n)$ with percentages (\%). The area under the curve (AUC) of receiver operating characteristic (ROC) was used to evaluate the discrimination ability of the mean, lowest, and highest levels of electrolytes and the variability of them. In order to assess the relationship between the mean, lowest, and highest levels of potassium, sodium, and chloride and in-hospital mortality, line charts were calculated and presented in three indices of variability. To test whether a trend across quartiles of three indices of variability of potassium, sodium, and chloride existed for risk estimates and to explore the relationship between the number of parameters with high variability and the inhospital mortality, logistic regression was used to calculate the odds ratios (ORs) and 95\% confidence intervals (CIs). Age, sex, diagnoses of disease, and surgical treatment were adjusted in the multivariable-adjusted logistic regression model 1 , while model 2 was further adjusted for mean levels of potassium, sodium, and chloride. Subgroup analyses were planned to be performed to evaluate whether the observed association of the number of parameters with high variability with the in-hospital mortality was modified by age, sex, or surgical treatment. $P<0.05$ was considered to indicate statistical significance.

Data extraction was conducted using PostgreSQL. All statistical analyses were performed using Stata 15.1 software and R 3.61 software.

\section{RESULTS}

\section{Study Subject Characteristics}

According to the eligibility criteria and exclusion criteria, a total of 11,245 children were included. The median (P25, P75) potassium, sodium, and chloride of all patients were 3.8 (3.58, 4.09), 136.83 (135.11, 138.60), and 108.67 (105.71, 111.17), respectively. The detailed mean values of these electrolytes in each group are shown in Table 1 and Supplementary Table 2. There were 5,916, 2,935, 1,681, and 713 children having $0,1,2$, or 3 parameters with high variability measured by $\mathrm{CV}$, respectively. Children's baseline characteristics by the number of parameters with high variability measured by $\mathrm{CV}$ are shown in Table 1. Patients who had more parameters with high variability had lower proportion of surgical experience. They also had higher variability in potassium, sodium, and chloride. Furthermore, these patients had longer length of PICU stay and higher inhospital mortality. Similar subject characteristics were observed when the variability was calculated as the SD and VIM (see Supplementary Tables 1, 2).

\section{The Association Between Electrolytes and the In-Hospital Mortality}

Line charts were conducted to assess the relationship between the mean, lowest, and highest levels of potassium, sodium, and chloride and the in-hospital mortality. To conclude, U-shaped relationships were observed. The lowest mortality was noted when serum potassium, sodium, and chloride was between $\sim 3.5$ and 5.0, 135 and 145, and 105 and $115 \mathrm{mmol} / \mathrm{l}$, respectively (see Supplementary Figure 1).

The ROCs of the mean, lowest, and highest levels of electrolytes and the variability of them were plotted for inhospital mortality (see Supplementary Figure 2). For potassium, although the highest levels of potassium had a good performance [OR (95\% CI): $0.753(0.732-0.774)]$, the three indices of variability showed larger AUCs [CV: 0.729 (95\% CI 0.708-0.749), SD: 0.743 (95\% CI 0.723-0.763), and VIM: 0.725 (95\% CI 0.704$0.745)$ ] than the mean [OR (95\% CI): $0.595(0.569-0.621)]$ and lowest [OR (95\% CI): $0.610(0.584-0.635)]$ levels of potassium in predicting the in-hospital mortality. For sodium, the three indices of variability showed larger AUCs [CV: 0.707 (95\% CI 0.685-0.728), SD: 0.704 (95\% CI 0.683-0.726), and VIM: 0.707 (95\% CI 0.661-0.705)] than the mean [OR (95\% CI): 0.515 (0.486-0.543)], lowest [OR (95\% CI): 0.633 (0.608-0.658)], and highest [OR (95\% CI): $0.652(0.626-0.678)]$ levels of sodium in predicting the in-hospital mortality. For chloride, the three indices of variability showed larger AUCs [CV: 0.710 (95\% CI 0.689-0.732), SD: 0.698 (95\% CI 0.676-0.720), and VIM: 0.683 (95\% CI 0.668-0.829)] than the mean [OR (95\% CI): 0.638 (0.611-0.665)] and highest [OR (95\% CI): 0.533 (505-0.561)] levels of chloride in predicting the in-hospital mortality, though the lowest levels of chloride showed a good performance [OR (95\% CI): $0.721(0.700-0.743)]$.

\section{Variability of Individual Parameters and the In-Hospital Mortality}

An incrementally higher in-hospital mortality was observed with the highest CV quartile of potassium, sodium, and chloride compared to the lowest quartile groups, unadjusted [OR (95\% $\mathrm{CI})$ : Q4 of $\mathrm{K}^{+}, 13.39$ (6.45-27.81); Q4 of $\mathrm{Na}^{+}, 6.03$ (3.66-9.94); Q4 of $\left.\mathrm{Cl}^{-}, 4.24(2.71-6.64)\right]$; adjusted for age, sex, different 
TABLE 1 | Characteristics of subjects according to number of parameters with high variability measured as coefficient of variation.

\begin{tabular}{|c|c|c|c|c|}
\hline & $\begin{array}{c}\text { O parameter } \\
(n=5,916)\end{array}$ & $\begin{array}{c}1 \text { parameter } \\
(n=2,935)\end{array}$ & $\begin{array}{c}2 \text { parameters } \\
(n=1,681)\end{array}$ & $\begin{array}{c}3 \text { parameters } \\
(n=713)\end{array}$ \\
\hline \multicolumn{5}{|l|}{ Demographic characteristics } \\
\hline Age (mean $\pm S D$, years old) & $2.86 \pm 3.76$ & $1.96 \pm 3.28$ & $2.03 \pm 3.4$ & $1.95 \pm 3.33$ \\
\hline Gender $\{$ male $[n(\%)]\}$ & $3,359(56.78)$ & 1,689 (57.55) & $961(57.17)$ & $427(59.89)$ \\
\hline \multicolumn{5}{|l|}{ Diagnoses of disease [n (\%)] } \\
\hline Diseases of the respiratory system & $554(9.36)$ & $300(10.22)$ & $147(8.74)$ & $69(9.68)$ \\
\hline Diseases of the nervous system & $388(6.56)$ & $180(6.13)$ & $136(8.09)$ & $56(7.85)$ \\
\hline Diseases of the circulatory system & $359(6.07)$ & $242(8.25)$ & $156(9.28)$ & $80(11.22)$ \\
\hline Diseases of the digestive system & $566(9.57)$ & $215(7.33)$ & $85(5.06)$ & $32(4.49)$ \\
\hline Diseases of the genitourinary system & $254(4.29)$ & $76(2.59)$ & $29(1.73)$ & $20(2.81)$ \\
\hline Diseases of the immune system & $178(3.01)$ & $60(2.04)$ & $31(1.84)$ & $26(3.65)$ \\
\hline Neoplasms & $291(4.92)$ & $81(2.76)$ & $72(4.28)$ & $31(4.35)$ \\
\hline Surgical treatment $[n(\%)]$ & $3,440(58.15)$ & $1,373(46.78)$ & $699(41.58)$ & $232(32.54)$ \\
\hline \multicolumn{5}{|l|}{ Potassium (mmol/l) } \\
\hline Mean & $3.85 \pm 0.37$ & $3.89 \pm 0.49$ & $3.86 \pm 0.55$ & $3.89 \pm 0.61$ \\
\hline SD & $0.38 \pm 0.13$ & $0.61 \pm 0.4$ & $0.67 \pm 0.47$ & $0.94 \pm 0.58$ \\
\hline VIM & $7.95 \pm 2.68$ & $12.37 \pm 5.82$ & $13.75 \pm 6.7$ & $19.06 \pm 8.92$ \\
\hline CV & $9.86 \pm 3.32$ & $15.38 \pm 7.43$ & $17.09 \pm 8.57$ & $23.71 \pm 11.41$ \\
\hline \multicolumn{5}{|l|}{ Sodium (mmol/l) } \\
\hline Mean & $136.88 \pm 2.43$ & $136.91 \pm 3.46$ & $137.25 \pm 5.71$ & $138.37 \pm 8.35$ \\
\hline SD & $2.52 \pm 0.89$ & $3.65 \pm 1.64$ & $5.62 \pm 2.61$ & $7.52 \pm 3.82$ \\
\hline VIM & $0 \pm 0$ & $0 \pm 0$ & $0 \pm 0$ & $0 \pm 0$ \\
\hline CV & $1.84 \pm 0.65$ & $2.67 \pm 1.19$ & $4.08 \pm 1.81$ & $5.38 \pm 2.49$ \\
\hline \multicolumn{5}{|l|}{ Chloride (mmol/l) } \\
\hline Mean & $108.8 \pm 3.59$ & $108.16 \pm 4.64$ & $107.52 \pm 6.43$ & $107.59 \pm 8.32$ \\
\hline SD & $2.91 \pm 1.06$ & $4.17 \pm 1.94$ & $6.12 \pm 2.53$ & $7.92 \pm 3.19$ \\
\hline VIM & $\begin{array}{c}2,7324.2 \pm \\
9,956.72\end{array}$ & $\begin{array}{c}38,955.26 \pm \\
18,196.28\end{array}$ & $\begin{array}{c}57,077.07 \pm \\
25,318.18\end{array}$ & $\begin{array}{c}74,377.23 \pm \\
33,739.14\end{array}$ \\
\hline CV & $2.68 \pm 0.98$ & $3.87 \pm 1.81$ & $5.7 \pm 2.28$ & $7.35 \pm 2.82$ \\
\hline Length of ICU stay (days) & $7.22 \pm 16.71$ & $11.07 \pm 18.66$ & $14.04 \pm 21.49$ & $16.87 \pm 23.07$ \\
\hline In-hospital death [n (\%)] & $101(1.71)$ & $197(6.71)$ & 216 (12.85) & $166(23.28)$ \\
\hline
\end{tabular}

Data were expressed as mean \pm standard deviation or $n(\%)$.

$\mathrm{CV}$, coefficient of variation; SD, standard deviation; VIM, variability independent of the mean.

diagnoses of disease, and surgery treatment (model 1) [OR (95\% $\mathrm{CI})$ : Q4 of $\mathrm{K}^{+}, 11.18$ (5.33-23.45); Q4 of $\mathrm{Na}^{+}, 4.74$ (2.84-7.91); Q4 of $\mathrm{Cl}^{-}, 3.01$ (1.89-4.80)]; and even further adjusted for mean levels of potassium, sodium, and chloride (model 2) [OR (95\% $\mathrm{CI})$ : Q4 of $\mathrm{K}^{+}, 10.12$ (4.78-21.42); Q4 of $\mathrm{Na}^{+}$, 4.63 (2.72-7.89); Q4 of $\mathrm{Cl}^{-}, 2.72$ (1.66-4.45)] (Table 2). Similar findings were noted when the variability was determined using the SD and VIM (all $p$ for trend $<0.001$ ) (see Supplementary Tables 3, 4).

\section{Number of Parameters With High Variability and the In-Hospital Mortality}

Furthermore, the composite effect of parameter variability on the in-hospital mortality was evaluated. An incrementally higher inhospital mortality was noted with a higher number of parameters with high variability (Table 3). After adjusting for possible confounding factors mentioned before, the ORs ( $95 \% \mathrm{CI}$ ) of inhospital mortality were 3.14 (2.44-4.04) for one parameter, 5.85 (4.54-7.53) for two parameters, and $10.32(7.81-13.64)$ for three parameters compared with children having no parameters of high variability measured as the $\mathrm{CV}$. The results were consistent when the variability was determined using the SD and VIM (all $P$ for trend $<0.001$ ).

To evaluate the influence of lower quartile groups together, a variability scoring system was defined. In the system, 0 point was assigned for Q1, 1 point for Q2, 2 points for Q3, and 3 points for Q4 groups for each of three parameters. Thus, the total score ranged from 0 to 9 . It was shown that there is a positive linear association between the variability score and the in-hospital mortality or OR for the in-hospital mortality (all $P$ for trend $<0.001$ ) (Figure 1).

\section{Subgroup Analysis}

Pre-specified stratified analyses by age, sex, and surgical treatment were conducted. The significant association between the number of parameters with high variability and the inhospital mortality was presented in all subgroups (Figure 2). 
TABLE 2 | Odds ratios and 95\% confidence intervals of in-hospital mortality by quartiles of electrolyte variability measured as coefficient of variation.

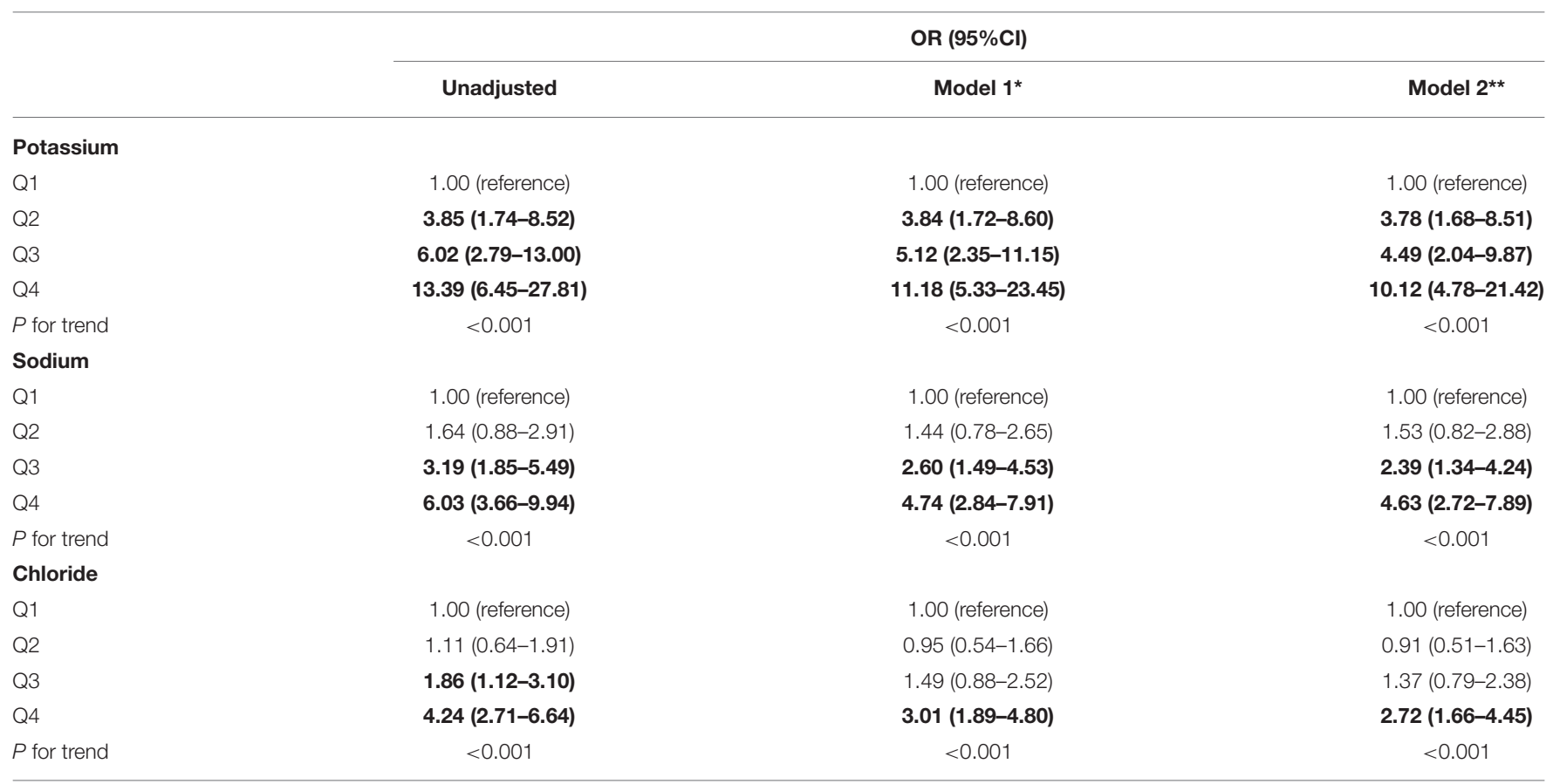

*Model 1 was adjusted for age, sex, diagnoses of disease, and surgical treatment.

**Model 2 was adjusted for model 1 plus mean values of electrolytes (potassium, sodium, and chloride).

Bold data are data with statistical significance.

TABLE 3 | Odds ratios and 95\% confidence intervals of in-hospital mortality by number of electrolytes with high variability.

\section{Number of electrolytes with high variability}

\begin{tabular}{c} 
\\
\hline Unadjusted \\
\\
1.00 (reference) \\
$\mathbf{4 . 1 4}(\mathbf{3 . 2 5 - 5 . 2 9 )}$ \\
$\mathbf{8 . 4 9}(\mathbf{6 . 6 6 - 1 0 . 8 3 )}$ \\
$\mathbf{1 7 . 4 7}(\mathbf{1 3 . 4 4 - 2 2 . 7 2 )}$ \\
$<0.001$
\end{tabular}

Measured as SD

0

1

2

3

$P$ for trend

\section{Measured as VIM}

\section{0}

1

2

3

$P$ for trend

\subsection{0 (reference) \\ 3.83 (3.01-4.87) \\ 8.17 (6.44-10.36) \\ 16.66 (12.77-21.72) \\ $<0.001$}

1.00 (reference)

3.72 (2.94-4.71)

7.32 (5.78-9.27)

15.18 (11.73-19.64)

$<0.001$

\section{OR $(95 \% \mathrm{Cl})$}

Model $1^{*}$

Model 2**
1.00 (reference)

3.14 (2.44-4.04)

5.85 (4.54-7.53)

10.32 (7.81-13.64) $<0.001$

1.00 (reference)

3.24 (2.53-4.14)

$6.60(5.17-8.43)$

11.65 (8.86-15.32)

$<0.001$

1.00 (reference)

2.87 (2.24-3.68)

5.44 (4.24-6.97)

8.83 (6.65-11.71)

$<0.001$

1.00 (reference)

3.14 (2.47-4.00)

5.98 (4.70-7.61)

11.45 (8.78-14.94)

$<0.001$
1.00 (reference)

2.93 (2.30-3.74)

5.50 (4.30-7.03)

9.63 (7.32-12.68) $<0.001$

*Model 1 was adjusted for age, sex, diagnoses of disease, and surgical treatment.

${ }^{\star \star}$ Model 2 was adjusted for model 1 plus mean values of electrolytes (potassium, sodium, and chloride).

$C V$, coefficient of variation; $S D$, standard deviation; VIM, variability independent of the mean.

Bold data are data with statistical significance. 


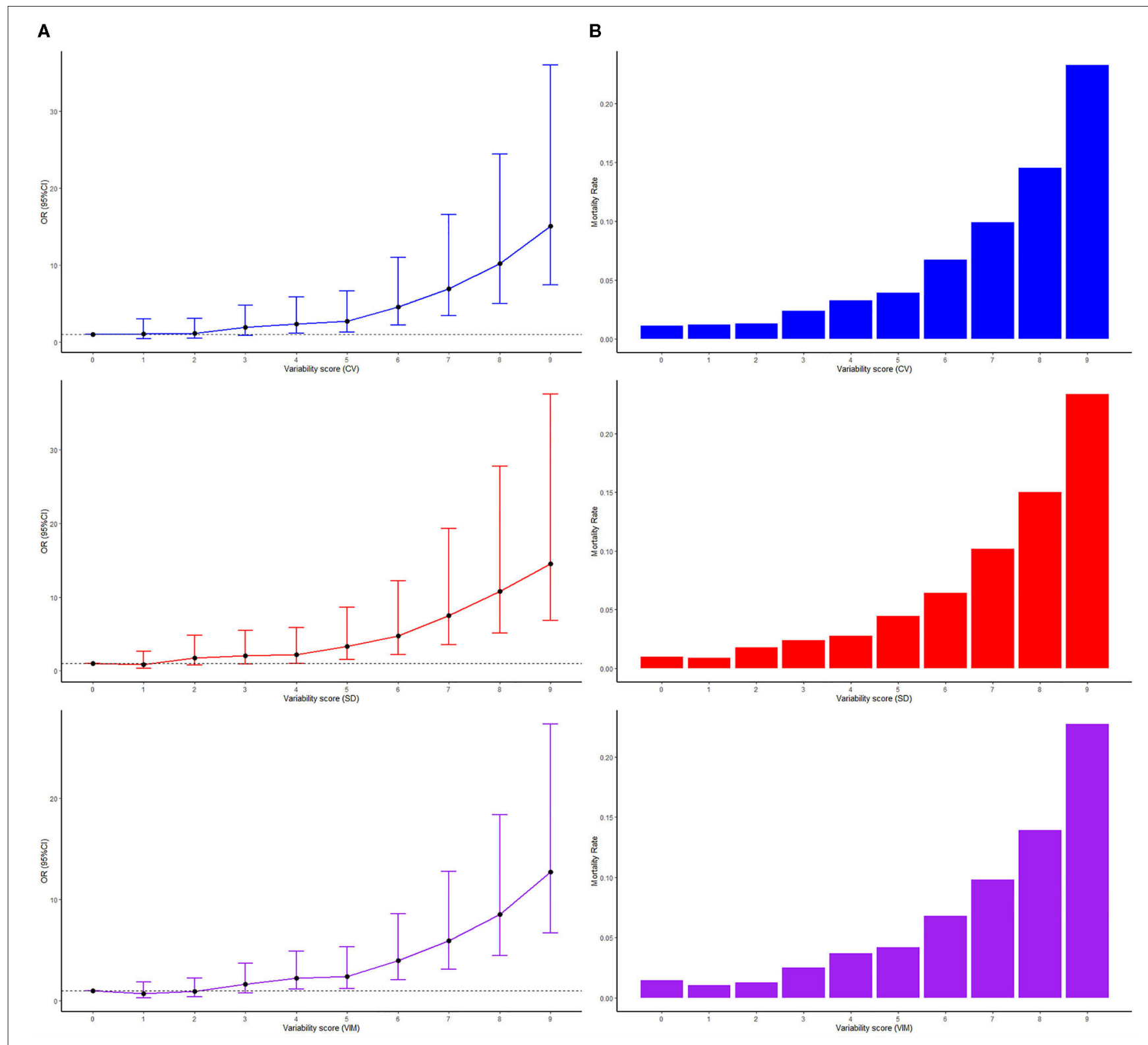

FIGURE 1 | A positive linear association between the variability score and (A) odds ratios and 95\% confidence interval for the in-hospital mortality (line charts) and (B) the in-hospital mortality (bar charts).

Higher adjusted ORs for the in-hospital mortality were observed in surgical patients (all $p$ for interaction $<0.001$ ), when the variability was determined using all of the three indices of variability. In addition, female subgroups showed higher inhospital mortality when the variability was determined using the $\mathrm{SD}$ ( $p$ for interaction $=0.038)$. However, other subgroups showed similar in-hospital mortality with high variability.

\section{DISCUSSION}

This study showed a U-shaped association between the level of serum potassium, sodium, and chloride and the in-hospital mortality of children in ICUs. We also examined the association between three indices of variability of these electrolytes and the in-hospital mortality. Individuals with higher variability of each parameter were at higher risk of in-hospital mortality. The results were consistent in different subgroups, promising that the relationship was widely applicable. Of note, the result of ROC showed that the variability was more sensitive and stable than the mean, lowest, and highest level of these parameters as risk factors of the in-hospital mortality in critically ill children admitted in ICUs.

In clinical practice, normal ranges of levels of serum potassium, sodium, and chloride are established mainly based on healthy population, and it is still unclear whether these values are also applicable to children because there has been few 


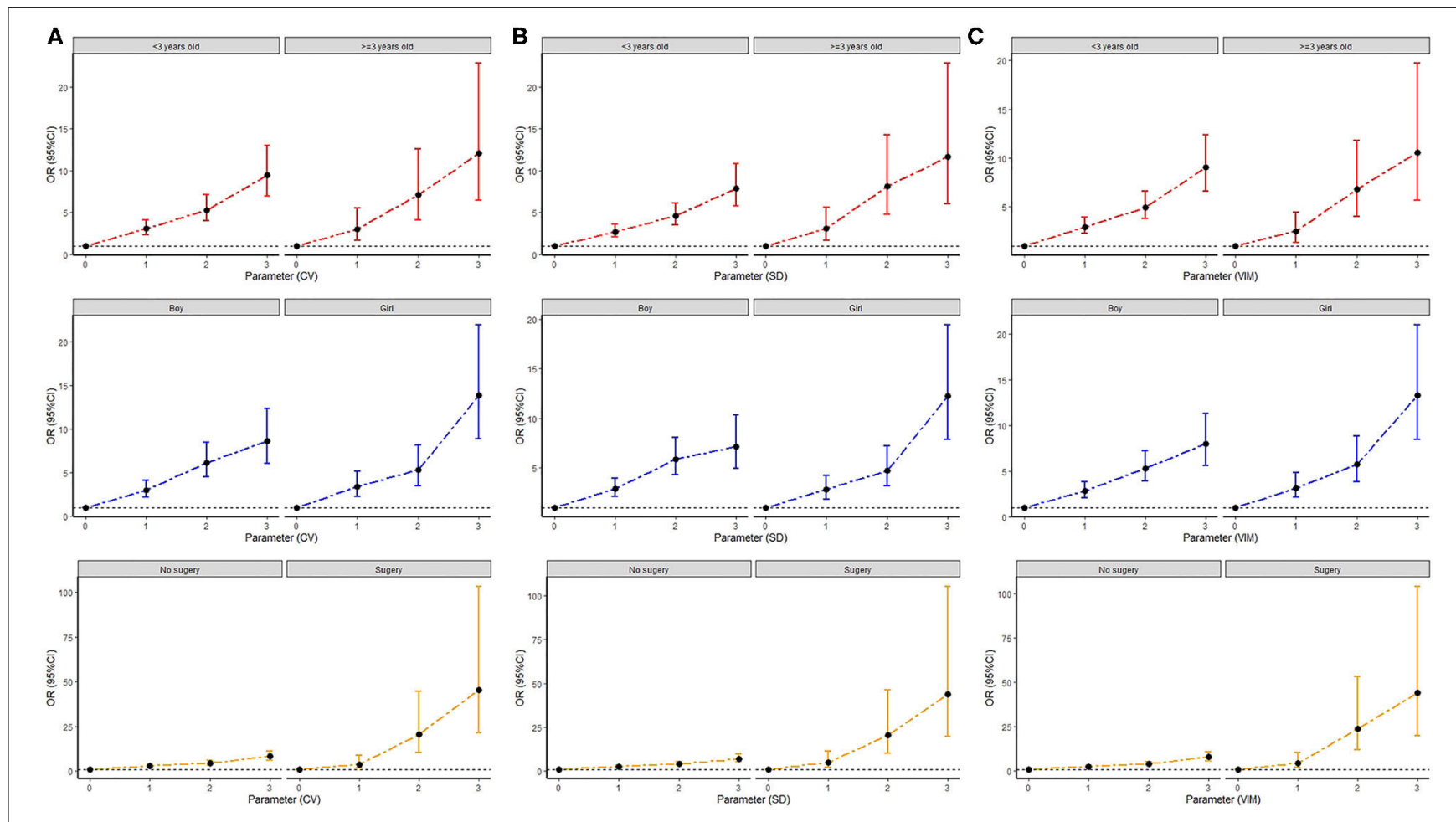

FIGURE 2 | The odds ratios and 95\% confidence interval for in-hospital mortality by number of parameters with high variability defined as the highest quartile of (A) CV of electrolytes; (B) SD of electrolytes; and (C) VIM of electrolytes. Subgroup analyses according to age, sex, and surgical experience. CV, coefficient of variation; $\mathrm{SD}$, standard deviation; VIM, variability independent of the mean.

evidence for clear recommendations on electrolyte management in critically ill children. We observed that children who had electrolytes in approximately normal ranges still had lower inhospital mortality. The lowest mortality was noted when serum potassium, sodium, and chloride was between 3.5 and 5.0, 135 and 145 , and 105 and $115 \mathrm{mmol} / \mathrm{l}$, respectively. However, the level of serum chloride in correlation with the lowest mortality differed from the normal range between 95 and $100 \mathrm{mmol} / \mathrm{l}$, indicating that the normal range of the electrolytes may not fit to all critically ill children. It is hard to make a regular summary for different levels of these parameters.

Recently, the variability in serum electrolytes has gained much attention as novel risk factors for in-hospital mortality in critically ill patients (19-21). Previous data suggested that the variability of potassium and sodium induced or reflected pathological conditions associated with mortality of critically ill patients, for it was related to membrane potential. Our results showed that the composite effect of potassium, sodium, and chloride variability was strongly related with the in-hospital mortality. Thus, the importance of stabilizing the variability of potassium, sodium, and chloride should be further emphasized. In this study, we observed that the variability of potassium, sodium, and chloride was more sensitive and stable in predicting the in-hospital mortality of critically ill children than the absolute levels of these parameters. Moreover, there was a positive linear relationship between the variability and the in-hospital mortality, which is more applicable in the clinical practice, compared to the level of these parameters. Considering that age and gender might affect the levels of electrolytes, and some studies showed that the imbalance in potassium and sodium may be related with early mortality following surgery $(22,23)$, we conducted the subgroup analyses to explore the relationship between variability indices and the in-hospital mortality in specific populations. As a result, higher adjusted ORs for in-hospital mortality were observed in the surgical group, which was consistent with previous studies $(22,23)$.

Critically ill children might have dysfunctions in organs that regulate electrolytes, such as the kidney, with consequent instability in several biological parameters, increasing the possibility of reverse causation. However, our data showed a strong correlation between variability indices and the in-hospital mortality with a dose-response relationship after adjusting potential confounding factors including the diagnosis of renal disease. The result was consistent with previous data, suggesting the electrolyte disturbance can directly increase the risk of mortality $(24,25)$. We infer that the variability in electrolytes is not only an indicator but also a risk factor for increased risk of severe health outcomes. We also performed subgroup analyses to strengthen the temporal relationship of the association and confirmed similar results. Our data suggested that the variability of these parameters is significantly related with the mortality, even in young children and other specific group population.

There were several strengths in this study. This is the first study, to our knowledge, exploring the association between 
different kinds of variability indices and mortality and compare the advantages and disadvantages between variability indices and the original values of electrolytes in critically ill children. The sample size of the present study was huge, allowing a relatively higher power to obtain the independent and joint prognostic effects of variability of serum potassium, sodium, and chloride. The most important point is that variability indices could reduce the bias caused by the treatment during the hospitalization, such as supplementation of potassium, sodium, and chloride.

There are limitations that should be noted. First, it was a retrospective analysis, and there might be discrepancies between the actual mortality and the information recorded in the database. Second, this study was a single-center study, and we could only be able to establish an association, rather than a causal relationship. Third, the different examining time points and possible inconsistency with different ways of measuring within the study period may influence the variability indices. Furthermore, it is possible that some of the unknown factors, such as genetic factors, may influence the mortality, although we tried to adjust covariates to minimize this possibility.

\section{CONCLUSIONS}

In summary, the effect of variability indices on predicting the inhospital mortality in critically ill children in ICUs was observed in this study. There was a linear association between the number of high variability parameters and the in-hospital mortality. However, larger-scale prospective studies are needed to validate the association.

\section{DATA AVAILABILITY STATEMENT}

Publicly available datasets were analyzed in this study. This data can be found here: https://www.nature.com/articles/s41597-0200355-4.

\section{REFERENCES}

1. Kraft MD, Btaiche IF, Sacks GS, Kudsk KA. Treatment of electrolyte disorders in adult patients in the intensive care unit. Am J Health Syst Pharm. (2005) 62:1663-82. doi: 10.2146/ajhp040300

2. Buckley MS, Leblanc JM, Cawley MJ. Electrolyte disturbances associated with commonly prescribed medications in the intensive care unit. Crit Care Med. (2010) 38 (6 Suppl.):S253-64. doi: 10.1097/CCM.0b013e3181dda0be

3. Gennari FJ. Disorders of potassium homeostasis: hypokalemia and hyperkalemia. Crit Care Clin. (2002) 18:27388. doi: 10.1016/S0749-0704(01)00009-4

4. Gumz ML, Rabinowitz L, Wingo CS. An integrated view of potassium homeostasis. N Engl J Med. (2015) 373:60-72. doi: 10.1056/NEJMra1313341

5. Torlén K, Kalantar-Zadeh K, Molnar MZ, Vashistha T, Mehrotra R. Serum potassium and cause-specific mortality in a large peritoneal dialysis cohort. Clin J Am Soc Nephrol. (2012) 7:1272-84. doi: 10.2215/CJN.00960112

6. McMahon GM, Mendu ML, Gibbons FK, Christopher KB. Association between hyperkalemia at critical care initiation and mortality. Intensive Care Med. (2012) 38:1834-42. doi: 10.1007/s00134-012-2636-7

7. Beseoglu K, Etminan N, Steiger HJ, Hänggi D. The relationship of early hypernatremia with clinical outcome in patients suffering from aneurysmal subarachnoid hemorrhage. Clin Neurol Neurosurg. (2014) 123:164-8. doi: 10.1016/j.clineuro.2014.05.022

\section{ETHICS STATEMENT}

The studies involving human participants were reviewed and approved by Institutional Review Board of the Children's Hospital, Zhejiang University School of Medicine (Hangzhou, China). Written informed consent from the participants' legal guardian/next of kin was not required to participate in this study in accordance with the national legislation and the institutional requirements.

\section{AUTHOR CONTRIBUTIONS}

JL conceptualized and designed the study, supervised data collection, carried out the initial analyses, and drafted the initial manuscript. JC and XT designed the data collection instruments and collected data. AS coordinated and supervised data collection, assisted in the statistical analysis, and carried out the initial analyses. JD and MC coordinated and supervised data collection and critically reviewed the manuscript for important intellectual content. YZ conceptualized and designed the study, supervised data collection, and reviewed and revised the manuscript. All authors have read and approved the final manuscript.

\section{ACKNOWLEDGMENTS}

The authors thank the Children's Hospital of Zhejiang University and Prof. Haomin Li for access to PIC's data.

\section{SUPPLEMENTARY MATERIAL}

The Supplementary Material for this article can be found online at: https://www.frontiersin.org/articles/10.3389/fped. 2021.692894/full\#supplementary-material

8. Bennani SL, Abouqal R, Zeggwagh AA, Madani N, Abidi K, Zekraoui A, et al. Incidence, causes and prognostic factors of hyponatremia in an intensive care unit. Rev Med Interne. (2003) 24:224-9. doi: 10.1016/S0248-8663(02)0 0811-1

9. Polderman KH, Schreuder WO, van Schijndel RJ, Thijs LG. Hypernatremia in the intensive care unit: an indicator of quality of care? Crit Care Med. (1999) 27:1105-8. doi: 10.1097/00003246-199906000-00029

10. Testani JM, Hanberg JS, Arroyo JP, Brisco MA, Ter Maaten JM, Wilson FP, et al. Hypochloraemia is strongly and independently associated with mortality in patients with chronic heart failure. Eur J Heart Fail. (2016) 18:660-8. doi: 10.1002/ejhf.477

11. Lee JY, Hong TH, Lee KW, Jung MJ, Lee JG, Lee SH. Hyperchloremia is associated with 30-day mortality in major trauma patients: a retrospective observational study. Scand J Trauma Resusc Emerg Med. (2016) 24:117. doi: 10.1186/s13049-016-0311-7

12. Emrath ET, Fortenberry JD, Travers C, McCracken CE, Hebbar KB. Resuscitation with balanced fluids is associated with improved survival in pediatric severe sepsis. Crit Care Med. (2017) 45:1177-83. doi: 10.1097/CCM.0000000000002365

13. Gosmanova EO, Mikkelsen MK, Molnar MZ, Lu JL, Yessayan LT, KalantarZadeh K, et al. Association of systolic blood pressure variability with mortality, coronary heart disease, stroke, and renal disease. J Am Coll Cardiol. (2016) 68:1375-86. doi: 10.1016/j.jacc.2016.06.054 
14. Bangalore S, Breazna A, DeMicco DA, Wun CC, Messerli FH, TNT Steering Committee and Investigators. Visit-to-visit lowdensity lipoprotein cholesterol variability and risk of cardiovascular outcomes: insights from the TNT trial. J Am Coll Cardiol. (2015) 65:1539-48. doi: 10.1016/j.jacc.2015.02.017

15. Bangalore S, Fayyad R, Laskey R, DeMicco DA, Messerli FH, Waters DD. Body-weight fluctuations and outcomes in coronary disease. $N$ Engl J Med. (2017) 376:1332-40. doi: 10.1056/NEJMoa1606148

16. Zeng X, Yu G, Lu Y, Tan L, Wu X, Shi S, et al. PIC, a paediatric-specific intensive care database. Sci Data. (2020) 7:14. doi: 10.1007/978-981-15-7981-3

17. Little J, Higgins JP, Ioannidis JP, Moher D, Gagnon F, von Elm E, et al. STrengthening the REporting of Genetic Association studies (STREGA): an extension of the STROBE statement. Ann Intern Med. (2009) 150:20615. doi: 10.7326/0003-4819-150-3-200902030-00011

18. Lee SH, Han K, Kwon HS, Yoon KH, Kim MK. Effect of variability in blood pressure, glucose and cholesterol concentrations, and body weight on emergency hospitalization and 30-day mortality in the general population. J Am Heart Assoc. (2020) 9:e017475. doi: 10.1161/JAHA.120.0 17475

19. Engelhardt LJ, Balzer F, Müller MC, Grunow JJ, Spies CD, Christopher $\mathrm{KB}$, et al. Association between potassium concentrations, variability and supplementation, and in-hospital mortality in ICU patients: a retrospective analysis. Ann Intensive Care. (2019) 9:100. doi: 10.1186/s13613-0190573-0

20. Bales J, Cho S, Tran TK, Korab GA, Khandelwal N, Spiekerman CF, et al. The effect of hyponatremia and sodium variability on outcomes in adults with aneurysmal subarachnoid hemorrhage. World Neurosurg. (2016) 96:3409. doi: 10.1016/j.wneu.2016.09.005

21. Hessels L, Hoekstra M, Mijzen LJ, Vogelzang M, Dieperink W, Lansink $\mathrm{AO}$, et al. The relationship between serum potassium, potassium variability and in-hospital mortality in critically ill patients and a before-after analysis on the impact of computer-assisted potassium control. Crit Care. (2015) 19:4. doi: 10.1186/s13054-014-0720-9
22. Shaw AD, Bagshaw SM, Goldstein SL, Scherer LA, Duan M, Schermer CR, et al. Major complications, mortality, and resource utilization after open abdominal surgery: 0.9\% saline compared to Plasma-Lyte. Ann Surg. (2012) 255:821-9. doi: 10.1097/SLA.0b013e31825074f5

23. Dawwas MF, Lewsey JD, Watson CJ, Gimson AE, UK, Ireland Liver Transplant Audit. The impact of serum potassium concentration on mortality after liver transplantation: a cohort multicenter study. Transplantation. (2009) 88:402-10. doi: 10.1097/TP.0b013e3181aed8e4

24. Siscovick DS, Raghunathan TE, Psaty BM, Koepsell TD, Wicklund KG, Lin X, et al. Diuretic therapy for hypertension and the risk of primary cardiac arrest. N Engl J Med. (1994) 330:1852-7. doi: 10.1056/NEJM199406303302603

25. Ioannou GN. Development and validation of a model predicting graft survival after liver transplantation. Liver Transpl. (2006) 12:1594-606. doi: 10.1002/lt.20764

Conflict of Interest: The authors declare that the research was conducted in the absence of any commercial or financial relationships that could be construed as a potential conflict of interest.

Publisher's Note: All claims expressed in this article are solely those of the authors and do not necessarily represent those of their affiliated organizations, or those of the publisher, the editors and the reviewers. Any product that may be evaluated in this article, or claim that may be made by its manufacturer, is not guaranteed or endorsed by the publisher.

Copyright (C) 2021 Lin, Zhang, Chen, Dai, Song, Chen and Tao. This is an open-access article distributed under the terms of the Creative Commons Attribution License (CC $B Y)$. The use, distribution or reproduction in other forums is permitted, provided the original author(s) and the copyright owner(s) are credited and that the original publication in this journal is cited, in accordance with accepted academic practice. No use, distribution or reproduction is permitted which does not comply with these terms. 